\title{
Monte Carlo study of a vortex glass model
}

\author{
J. D. Reger ${ }^{1}$ and A. P. Young ${ }^{2}$ \\ 1 Institut für Physik, Universität Mainz, D-6500 Mainz, Germany \\ and AIX Competence Center, IBM Deutschland GmbH, D-8000 Munich, Germany \\ 2 Physics Department, University of California Santa Cruz, Santa Cruz, CA 95064.
}

\begin{abstract}
We describe results of Monte Carlo simulations on a model that seems to have the necessary ingredients to describe a disordered type-II superconductor in a magnetic field. We compute the free energy cost to twist the direction of the phase of the condensate and analyze the results by finite-size scaling. The results show convincingly that the model has different behavior as a function of dimension: in $d=4$ the model clearly has a finite transition temperature; $T_{c}$, while for $d=2$ only there is only a transition at $T=0$.
\end{abstract}

Submitted to J. Phys. A. 
Since fluctuation effects play a much more important role in high temperature superconductors than in conventional superconducting materials, there has been a great deal of effort [1] to understand the behavior of type II superconductors in a magnetic field, including the effects of disorder, when one goes beyond the mean field picture of BCS or Ginzburg-Landau theories. One intriguing aspect which has emerged is the possibility of a vortex glass phase [2, 3] in which the off diagonal long range order of the pair condensate has a phase which is random in space but frozen in time, much like the order parameter in a spin glass $\llbracket$. This can arise because the Abrikosov flux lattice, which forms in pure samples, is destroyed by disorder in less than 4 dimensions [5] beyond a certain length scale, $l_{d i s}$. The phase of the condensate does not then form a regular periodic pattern on scales larger than $l_{d i s}$, but, according to the vortex glass hypothesis, the system undergoes a transition into a spin glasslike state in which the phase is frozen in time. At the transition, the vortex glass correlation length, $\xi$ diverges. A number of experiments [6] have found evidence for such a transition in the $I-V$ characteristics of $\mathrm{Y}-\mathrm{B}-\mathrm{Cu}-\mathrm{O}$ samples. Only if there is a vortex glass phase does the resistance really vanish [1] for $H>H_{c_{1}}$. Otherwise, the resistance is, in principle, finite because clusters of vortices on scale $\xi$ can move by thermal activation over barriers, a process known as "flux creep" [7]. These effects are observable [8] in high- $T_{c}$ compounds since they have much larger fluctuations than conventional materials.

In this paper we descrive results of Monte Carlo simulations, analyzed by finitesize-scaling techniques, on a model that seems to have the necessary ingredients to describe the vortex glass state. The results show convincingly the different behavior occurs in different dimensions. In $d=4$ there is clearly a transition at finite transition temperature $T_{c}$ with vortex glass order at lower temperature, while in $d=2$ there is only a transition at $T=0$. Analogous results for $d=3$ have been presented before [9], and indicate behavior close to what is expected at the lower critical dimension, though with probably a finite $T_{c}$. Our results for $d=4$ have been briefly described 
in a conference proceeding [10]. We feel that it is useful to include them here as a contrast to the new results for $d=2$ to emphasise the power of the finite-size-scaling technique in elucidating whether or not a glass-like transition occurs.

The model that we study, known as the "gauge glass", has the following Hamiltonian:

$$
\mathcal{H}=-\sum_{<i, j>} \cos \left(\phi_{i}-\phi_{j}-A_{i j}\right)
$$

The phase, $\phi_{i}$, is defined on each site of a regular lattice, square for two dimensions, simple cubic for $d=3$ and simple hypercubic for $d=4$, with $N=L^{d}$ sites. Periodic boundary conditions are imposed. The sum is over all nearest neighbor pairs on the lattice. The effects of the magnetic field and disorder are represented by the quenched vector potentials, $A_{i j}$, which we take to be independent random variables with a uniform distribution between 0 and $2 \pi$. This model seems to be the simplest one with the correct ingredients of randomness, frustration and order parameter symmetry. It does, however, ignore screening, and therefore corresponds to an extreme type II limit in which $\kappa=\lambda / \xi \rightarrow \infty$, where $\lambda$ is the penetration length. Since $\kappa \gg 1$ in the high $T_{c}$ superconductors, this limit is not unreasonable. It is unclear, however, how much inclusion of screening via a fluctuating gauge field would modify the behavior of Eq. (1).

If the $A_{i j}$ are restricted to the values 0 and $\pi$, the model becomes the $X Y$ spin glass, for which the lower critical dimension is believed [11] to be 4. However, earlier work [13, 12, 9], has shown that the gauge glass is in a different universality class from the $X Y$ spin glass, presumably because it does not have the the "reflection" symmetry, $\phi_{i} \rightarrow-\phi_{i} \forall i$ 13.

As discussed before [9, 12], it is useful to consider the change in free energy $\Delta F$ when one imposes a twist $\Theta$ along one of the space directions, $x$ say. More precisely, the periodic boundary conditions, $\phi_{i}=\phi_{i+L \hat{x}}$ are replaced by the twisted boundary conditions, $\phi_{i}=\phi_{i+L \hat{x}}+\Theta$. By a simple redefinition of the phases $\phi_{i}$ one can replace this situation by a system with periodic boundary conditions and an extra 
contribution, $\Theta / L$, to the vector potential on bonds in the $x$-direction.

By Monte Carlo methods one can calculate derivatives of the free energy w.r.t. $\Theta$, so, for a single sample, we define a current, $I$, and a stiffness, $Y$, by

$$
\begin{gathered}
I \equiv \frac{\partial F}{\partial \Theta}=\frac{1}{L} \sum_{i}\left\langle\sin \Delta_{i}\right\rangle_{T} \\
Y \equiv \frac{\partial^{2} F}{\partial \Theta^{2}}=\frac{1}{L^{2}}\left\{\sum_{i}\left\langle\cos \Delta_{i}\right\rangle_{T}-\frac{1}{T} \sum_{i, j}\left[\left\langle\sin \Delta_{i} \sin \Delta_{j}\right\rangle_{T}-\left\langle\sin \Delta_{i}\right\rangle_{T}\left\langle\sin \Delta_{j}\right\rangle_{T}\right]\right\},
\end{gathered}
$$

where $\Delta_{i}=\phi_{i}-\phi_{i+\hat{x}}-A_{i, i+\hat{x}}, F$ is the total free energy and $i+\hat{x}$ refers to the nearest neighbor site in the $x$-direction from $i$. Note that both $I$ and $Y$ are gauge invariant so they are still useful even if one includes fluctuating gauge fields.

Above $T_{c}, \Delta F$, and hence both $I$ and $Y$, go to zero rapidly with increasing system size because the system is insensitive to boundary conditions when $L$ is much greater than the vortex glass correlation length $\xi$. If $T_{c}$ is finite, then, below $T_{c}, I$ and $Y$ vary with $L$ as $L^{\theta}$ where $\theta(>0)$, is an exponent describing the low temperature phase. In other words, $I$ and $Y$ increase with increasing $L$ below $T_{c}$, the opposite of what happens above $T_{c}$. Precisely at $T_{c}$, both $I$ and $Y$ are independent of size. Hence if $T_{c}$ is finite, $I$ and $Y$ should come together at $T_{c}$ and splay out again at lower temperatures. By contrast, if $T_{c}=0$, then, at $T=0, I$ and $Y$ vary as $L^{\theta}$ but with $\theta<0$. Consequently, $I$ and $Y$ decrease with $L$ even at $T=0$.

In a disordered system, it is necessary to perform an average over different realizations of the disorder, which we indicate by $[\cdots]_{\mathrm{av}}$. For the gauge glass, the average values of $I$ and $Y$ are both zero, i.e.

$$
[Y]_{\mathrm{av}}=[I]_{\mathrm{av}}=0
$$

because the configuration in which the vector potentials in the $x$ direction have been increased by $\Theta / L$ has the same weight in the configurational average as the original choice of vector potentials. One is therefore interested in the root mean square fluctutation between samples. This means that many samples must be averaged over, 
typically several thousand. If $T_{c}$ is finite, the finite size scaling form for the r.m.s. current, $\Delta I$ is therefore

$$
\Delta I \equiv\left[I^{2}\right]_{\mathrm{av}}^{1 / 2}=\tilde{I}\left(L^{1 / \nu}\left(T-T_{c}\right)\right) \quad\left(T_{c}>0\right)
$$

where $\nu$ is the correlation length exponent. We shall concentrate on the r.m.s. current in what follows, rather than the stiffness, because sample to sample flucutations in the stiffness have an asymmetric distribution with a long tail, which makes it difficult to get good statistics [9]. If $T_{c}=0$ then $\Delta I$ decreases with size even at $T=0$, i.e. $\Delta I \sim L^{\theta}$ where $\theta$ is negative and related to the exponent $\nu$ giving the divergence of the correlation length as $T \rightarrow 0$ by $-\theta=1 / \nu$. The finite size scaling form is then

$$
L^{1 / \nu} \Delta I=\tilde{I}\left(L^{1 / \nu} T\right) \quad\left(T_{c}=0\right)
$$

Tests to ensure equilibration were carried out as described elsewhere [14].

We first show results for $d=4,[10$. Fig. 1 shows clearly that the data for the r.m.s. current for different sizes come together at $T=T_{c} \simeq 0.95$ and then splay out again on the low temperature side, just as expected at a finite temperature transition. This provides unambigous evidence that there is a vortex glass transition in four dimensions (and presumably also in higher dimensions) with vortex-glass order on the low-temperature side. A scaling plot corresponding to Eq. (5) is shown in Fig. 2. From the fit we estimate

$$
T_{c}=0.96 \pm 0.01, \quad \nu=0.7 \pm 0.15 \quad(d=4)
$$

Next we discuss the case of $d=2$. The results for $\Delta I$ are shown in Fig. 3. Notice that they are quite different from Fig. 1, since, even at the lowest temperature, $\Delta I$ decreases with increasing size. This is precisely what is expected at a zero temperature transition, and the scaling plot in Fig. 4 corresponding to Eq. (6) works very well. From the fit we estimate

$$
T_{c}=0, \quad \nu=2.2 \pm 0.2 \quad(d=2)
$$


The value for $\nu$ agrees with earlier work [12], in which a different finite-size-scaling technique was used. Recent experiments [15] on very thin (16̊) films of YBCO have provided striking confirmation that $T_{c}=0$ for the vortex glass in two dimensions. Furthermore, it is found that non-linear current-voltage characteristics set in when the current density exceeds a value, $J_{n l}$ which, according to scaling theory [12] varies with temperature as $T^{1+\nu}$. According to conventional flux creep theory, there is no divergent length scale as $T \rightarrow 0$, which corresponds to setting $\nu=0$. The experiments 15] find $1+\nu=3.0 \pm 0.3$ in excellent agreement with the results presented here and in Ref. [12, but in clear disagreement with the flux creep theory. We emphasize, then, that the vortex glass picture leads to measurable consequences even when $T_{c}=0$.

To conclude, we have shown that a Monte Carlo calculation of the change in free energy due to a twist in the boundary conditions combined with finite size scaling is a very powerful tool for systems with $X Y$-like symmetry. We have shown that it clearly distiguishes between the finite temperature transition in the $d=4$ gauge glass and the zero temperature transition of the two-dimensional model.

Acknowledgments: The work of APY was supported in part by the NSF grant No. DMR 91-11576. The work of JDR was supported in part by the the Deutsche Forschungsgesellschaft through Sonderforschungsbereich 262/D1. Parts of the computations were performed on the Cray-YMP 8/832 at the German Supercomputer Center (HLRZ), Jülich. It is a pleasure to acknowledge M. P. A. Fisher for informative discussions. 


\section{References}

[1] D. S. Fisher, M. P. A. Fisher and D. A. Huse Phys. Rev. B 43, 130 (1991).

[2] M. P. A. Fisher, Phys. Rev. Lett. 62, 1415 (1989).

[3] W. Y. Shih, C. Ebner and D. Stroud, Phys. Rev. B 30, 134 (1984); S. John and T. C. Lubensky Phys. Rev. B 34, 4815 (1986).

[4] K. Binder and A. P. Young, Rev. Mod. Phys. 58, 801 (1986).

[5] A. I. Larkin, Sov. Phys. JETP 31, 784 (1970); A. I. Larkin and Yu. N. Ovchinikov, J. Low. Temp. Phys. 34, 409 (1979).

[6] R. H. Koch, V. Foglietti, W. J. Gallagher, G. Koren, A. Gupta and M. P. A. Fisher, Phys. Rev. Lett. 63, 1511 (1989); P. L. Gammel, L. F. Schneemener and D. J. Bishop, Phys. Rev. Lett. 66, 953 (1991); H. K. Olsson, R. H. Koch, W. Eidelloth and R. P. Robertazzi, Phys. Rev. Lett. 66, 2661 (1991).

[7] See e. g. Y. B. Kim and M. J. Stephen, in Superconductivity, R. D. Parks, ed. (Dekker, New York, 1969), vol. 2.

[8] M. Tinkham, Phys. Rev. Lett. 61, 1658 (1988); A. P. Malozemoff in Physical Properties of High Temperature Superconductors I, D.M. Ginsberg, ed., World Scientific, Singapore, (1989).

[9] J. D. Reger, T. A. Tokuyasu, A. P. Young and M. P. A. Fisher, Phys. Rev. B 44, 7147 (1991).

[10] J. D. Reger, in Computer Simulation Studies in Condensed Matter Physics V., ed. by D.P. Landau, K.K. Mon and H.-B. Schüttler, Springer-Verlag, Berlin, 1992. 
[11] S. Jain and A. P. Young, J. Phys. C 19, 3913 (1986); B. W. Morris, S. G. Colborne, M. A. Moore, A. J. Bray and J. Canisius, J. Phys. C 19, 1157 (1986); J. R. Banavar and M. Cieplak, Phys. Rev. Lett. 48, 832 (1982).

[12] M. P. A. Fisher, T. A. Tokuyasu and A. P. Young Phys. Rev. Lett. 66, 2931 (1991).

[13] D. A. Huse and H. S. Seung, Phys. Rev. B 42, 1059 (1990).

[14] R. N. Bhatt and A. P. Young, Phys. Rev. B 37, 5606 (1988); R. N. Bhatt and A. P. Young, Phys. Rev. Lett. 54, 924 (1985).

[15] C. Dekker, P. J. M. Wöltgens, R. H. Koch. B. W. Hussey and A. Gupta, Phys. Rev. Lett. 69, 2717 (1992). 


\section{Figure Captions}

Figure 1: The r.m.s. current, $\Delta I=\left[I^{2}\right]_{a v}^{1 / 2}$, for $d=4$ determined by Monte Carlo simulations for different sizes and temperatures. The curves for different sizes are expected to come together at $T_{c}$ and, if there is order in the low temperature state, to splay out again at lower temperatures. The data does indeed follow this behavior.

Figure 2: The same data as in Figure 1 but in a finite size scaling plot, with $T_{c}=0.96$ and $\nu=0.7$.

Figure 3: The r.m.s. current, $\Delta I=\left[I^{2}\right]_{a v}^{1 / 2}$, for $d=2$ determined by Monte Carlo simulations for different sizes and temperatures. The curves for different sizes do not come together, even at the lowest temperature. This behavior indicates a transition at $T=0$.

Figure 4: The same data as in Figure 4 but in a finite size scaling plot, with $T_{c}=0$ and $\nu=2.2$. 


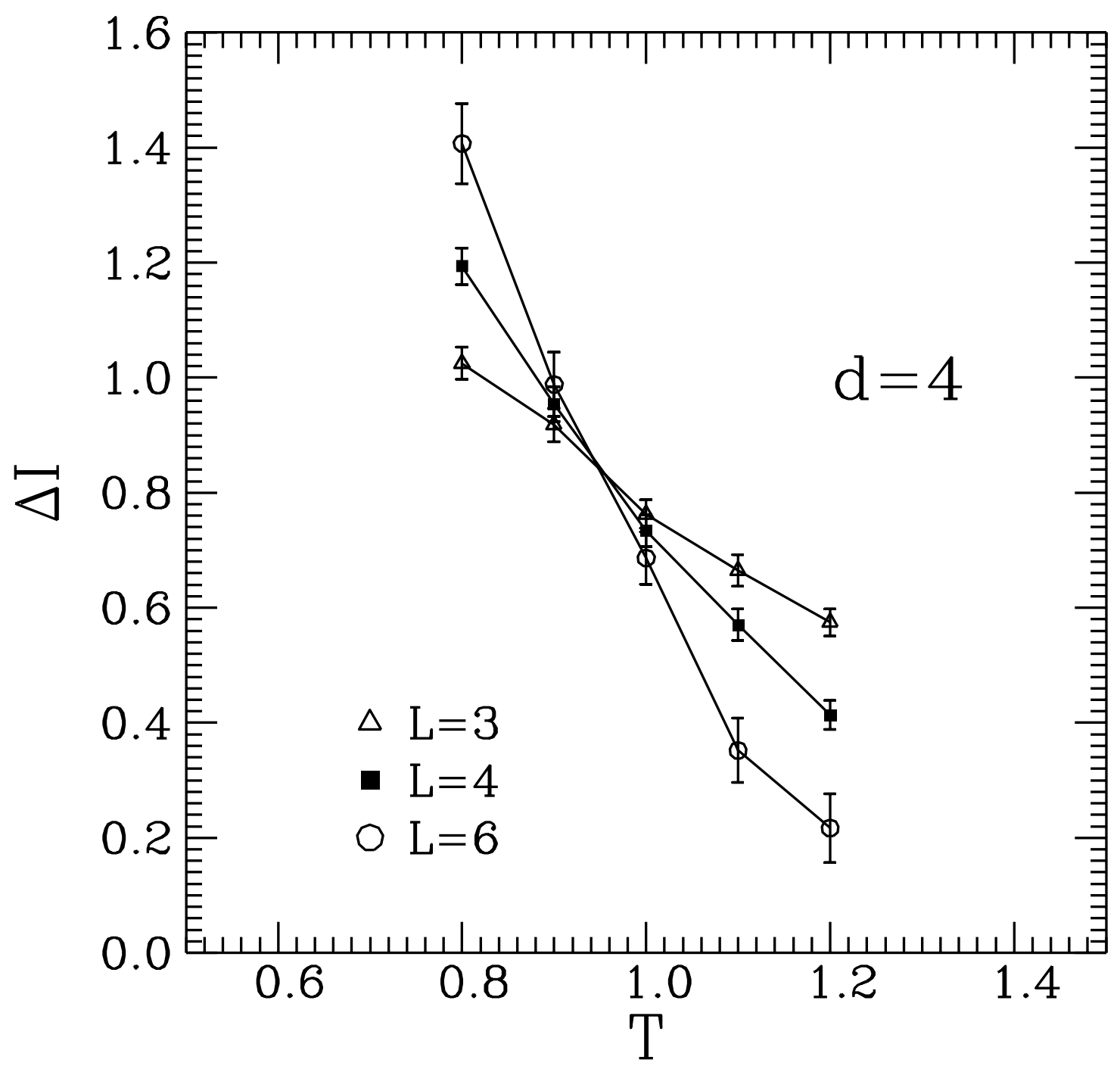

Figure 1 


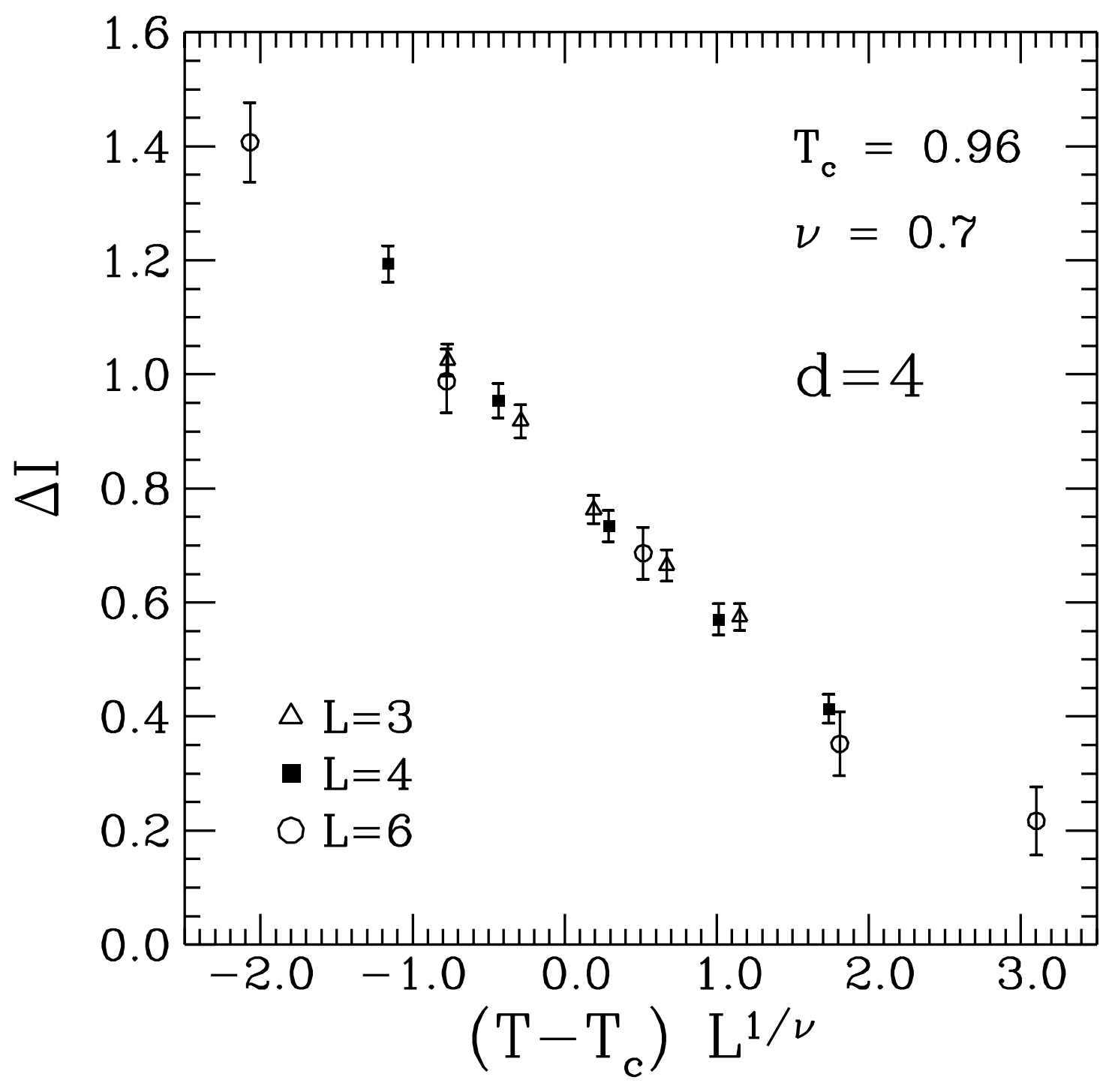

Figure 2 


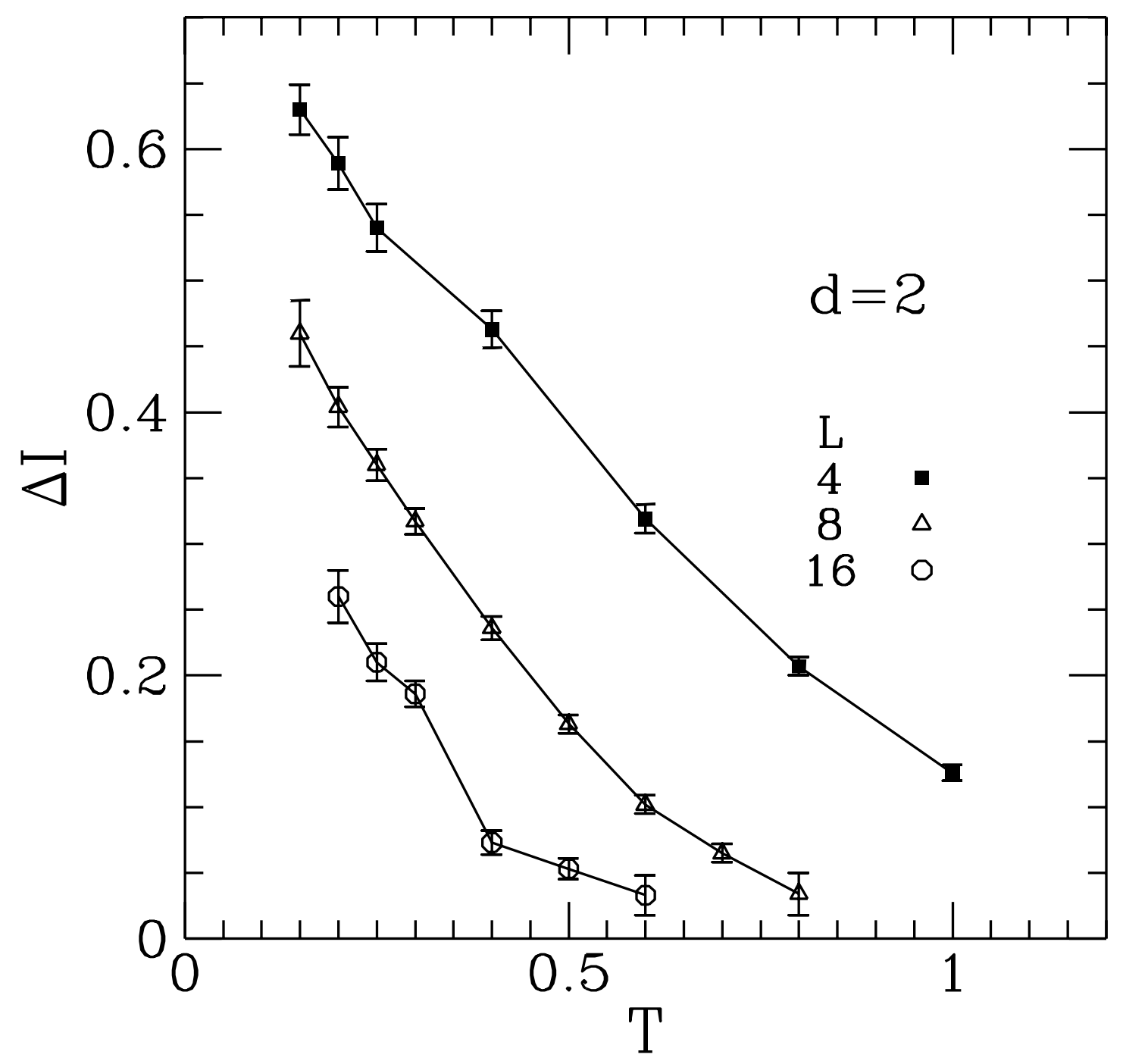

Figure 3 


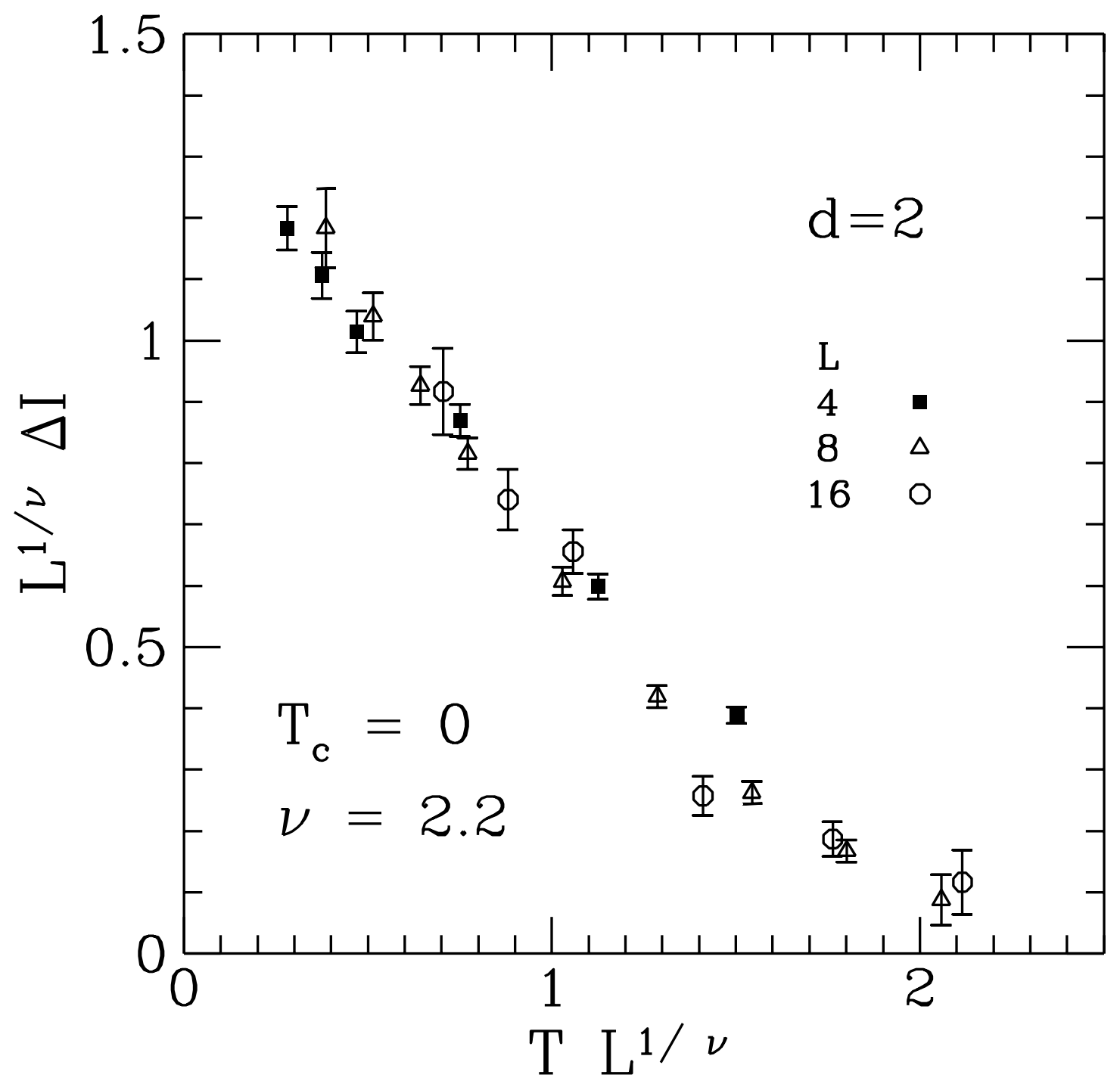

Figure 4 